Rev. Elev. Méd. vét. Pays trop., 1974, 27 (4) : 403-418

\title{
La brucellose en Afrique occidentale et son incidence sur la santé publique. Résultats de dix enquêtes épidémiologiques effectuées en Côte d'Ivoire, Haute-Volta et Niger, de 1970 à 1973
}

\author{
par R. GIDEL (*), J.-P. ALBERT $(* *)$, G. LE MAO (**) \\ et M. RETIF $(* * *)$ \\ (avec la collaboration technique de B. ATHAWET $\left({ }^{* * * *}\right)$, A. CISSE $(* * * *)$, \\ M. SIMPORE (****) et du personnel de la Section Biologie)

\section{RESUME} \\ Utilisant ring-tests, réactions allergologiques et sérologiques, les \\ auteurs ont étudié l'épidémiologie de la brucellose en Afrique de l'Ouest \\ au cours de dix enquêtes couplées, humaines et animales, où plus de \\ 120 villages ont été prospectés pàr sondage aléatoire. \\ Chez les animaux, 4108 ring-tests et 1225 sérologies et, chez l'homme, \\ 12739 intradermo-réactions et 11990 sérologies ont été effectuées. \\ La maladie animale existe partout et concerne les différentes espèces, \\ en particulier les bovins, mais à des taux très variables selon les régions, \\ le sud étant plus atteint que le nord. \\ Chez l'homme, la maladie intéresse essentiellement les populations \\ pastorales du sahel, tandis qu'ailleurs, elle affecte surtout les bergers et \\ leurs familles, les réactions allergologiques positives étant plus fréquentes \\ chez l'homme que chez la femme et chez l'adulte que chez l'enfant.
}

\section{INTRODUCTION}

La brucellose, dont l'extension est maintenant universelle, est bien connue des responsables sanitaires de l'Elevage car, dans de nombreux pays, elle représente pour ceux-ci un grave sujet de préoccupation.

Mais la brucellose n'est pas seulement une maladie vétérinaire. En effet, par son incidence importante en santé publique, elle est considérée, à juste titre, comme une zoonose majeure. Son rôle dans ce domaine a été souligné à maintes reprises, tant par l'Organisation Mondiale de la Santé (OMS) que par l'Organisation

O.C.C.G.E., Centre Muraz, Bobo-Dioulasso, République de Haute-Volta.

(*) Docteur vétérinaire.

(**) Docteurs en médecine.

$(* * *)$ Technicien de laboratoire.

$(* * * *)$ Infirmiers spécialistes. des Nations Unies pour l'Alimentation et l'Agriculture (FAO).

Cependant son importance en Afrique Tropicale semble avoir été sous-estimée, notamment en santé publique, alors que paradoxalement, les premiers cas de brucellose en Afrique de l'Ouest furent détectés chez l'homme dès le début du siècle. Dans le cadre de ses recherches sur l'épidémiologie des zoonoses et à la demande de plusieurs Etats membres de l'OCCGE (Organisation Commune contre les Grandes Endémies), le Centre Muraz a entrepris une série d'enquêtes couplées, humaines et animales, dans différentes zones bioclimatiques allant des régions sahéliennes du Niger et du Nord de la Haute-Volta, aux régions forestières littorales du Sud de la Côte d'Ivoire.

Le début de ce travail était d'étudier l'épidémiologie et l'importance de la brucellose chez différentes espèces animales et chez l'homme, ainsi que les relations existant entre les deux affections. 


\section{RAPPELS CLINIQUES SOMMAIRES}

\subsection{Généralités}

La brucellose est susceptible d'atteindre les différentes espèces animales, où elle évolue habituellement sous une forme chronique ou inapparente, mais ce sont les ruminants qui lui paient le plus lourd tribut. Autrefois surtout maladie de la chèvre et du mouton, elle est devenue, dans bien des régions du monde, un fléau de l'élevage bovin, et la brucellose bovine constitue maintenant une importante source d'infection pour l'homme.

Un des caractères de l'infection brucellique est qu'il n'existe aucune spécificité vis-à-vis des différentes espèces de Brucella. On retrouve ici les mêmes particularités que pour les bacilles tuberculeux. Bien que Brucella bovis ait une prédilection pour les grands ruminants, Brucella melitensis pour les petits ruminants et Brucella suis pour les pores, tous les animaux sont en fait sensibles à chacune des principales espèces, et l'homme ne fait pas exception.

\subsection{Chez les grands ruminants}

La forme génitale, caractérisée essentiellement par l'avortement chez les femelles, est considérée en général comme étant relativement rare en Afrique. En fait, lors de nos différentes enquêtes, les commémoratifs recueillis auprès des éleveurs ont révélé bien souvent l'existence de nombreux avortements dans toutes les régions où nos examens ont confirmé la fréquence de la maladie. Nous pensons plutôt, comme BEAUPERE (3), qu'en brousse, le contrôle des cas abortifs est extrêmement délicat en raison même du régime extensif de l'élevage et du nomadisme ou semi-nomadisme de la plupart des troupeaux dispersés dans une végétation qui réalise un véritable camouflage en saison des pluies. De surcroît, l'éleveur qui peut s'apercevoir de ces accidents hésitera, pour des raisons diverses, à les déclarer au service sanitaire, ne leur attribuant qu'une importance très secondaire.

Les localisations articulaires et synoviales, groupées sous le vocable général d'hygromas, peuvent apparaître en des points très variés du corps (bursites, arthrites, abcès sous-cutanés). Elles sont fréquentes en Afrique.

\subsection{Chez les petits ruminants}

La symptomatologie est particulièrement fruste et les formes chroniques ou asymptomatiques sont plus fréquentes que chez les bovins. Les avortements existent, mais leur évaluation est encore plus difficile que chez les grands ruminants. Il semblerait que la brucellose, même en l'absence d'avortements, soit un facteur de stérilité chez la chèvre et la brebis. Les porteurs chroniques de Brucella apparaissent nombreux et sont une source importante de contage.

\subsection{Chez l'homme}

La brucellose se caractérise avant tout par une symptomatologie très protéiforme. Les formes aiguës septicémiques, caractérisées par un début grippal avec fièvre et douleurs à type de névralgies, d'arthralgies et d'ostéoalgies sont relativement rares et plus fréquentes apparaissent les formes chroniques qui se manifestent fréquemment par des complications ostéoarticulaires pseudo-tuberculeuses.

L'image clinique variable et inconstante, les symptômes, aussi nombreux que divers, ne permettent pas un diagnostic clinique facile. Ceci explique que les cas réels sont infiniment plus nombreux que les cas déclarés et qu'il ne faille accorder qu'un crédit très limité aux données statistiques qui, lorsqu'elles existent, sont, la plupart du temps, fausses.

L'Organisation Mondiale de la Santé considère que la brucellose représente, dans le monde entier, une cause importante de morbidité, d'incapacité de travail et de réduction d'activité.

La brucellose humaine est une zoonose à cycle de transmission simple, tantôt direct, tantôt indirect, notamment par l'absorption de laitages infectés. Contrairement à la tuberculose, la transmission à l'homme constitue une impasse épidémiologique. La contamination interhumaine est en effet exceptionnelle. Quelques cas ont été cependant signalés, tels ceux relatant la transmission de la maladie par une mère allaitante à son nourrisson. Les réservoirs de virus animaux représentent donc en fait l'origine unique de la maladie chez l'homme. Cette dernière disparaît si on supprime la maladie animale. 
Les modes de transmission de la brucellose des animaux à l'homme sont le contact, l'ingestion, l'inhalation et l'inoculation. On considère la contamination par contact comme le mode de transmission le plus fréquent, ainsi qu'en témoigne le caractère souvent professionnel de l'affection chez l'homme. Il ne faut toutefois pas sous-estimer en Afrique le rôle de la transmission par le lait chez des populations pastorales pour lesquelles celui-ci constitue une des bases de l'alimentation et qui, de surcroît, est consommé très fréquemment à l'état cru.

La brucellose a donc une double incidence sur la santé de l'homme, directe par les troubles pathologiques variés qu'elle est susceptible de déterminer, et indirecte par les pertes en protéines d'origine animale qu'elle occasionne.

\section{REGIONS PROSPECTEES}

Dix enquêtes ont eu lieu au total et ont concerné trois Etats de l'Afrique de l'Ouest. Cinq enquêtes ont eu lieu en Côte d'Ivoire, quatre en Haute-Volta et une au Niger. Classées en fonction des données climatologiques, elles se répartissent ainsi :

\subsection{Savane sahélienne}

Deux enquêtes ont eu lieu en Haute-Volta (Dori et Markoye) et une enquête au Niger (région de Niamey). Ce sont des contrées à pluviométrie faible (300 à $500 \mathrm{~mm}$ par an), caractérisées par une longue saison sèche de 9 mois, fraîche d'octobre à février (refroidissement nocturne important), torride de mars à juin, et une saison des pluies de 3 mois (juillet à septembre). Le paysage est celui d'une steppe arborée à prédominance d'épineux se raréfiant du Sud au Nord, faiblement vallonnée, parsemée de dunes et de quelques affleurements rocheux avec un tapis herbacé discontinu. La nappe phréatique est profonde. Le réseau hydrographique est intermittent. La plupart des mares et marigots tarissent au cours de la longue saison sèche durant laquelle l'évaporation est importante ( $3 \mathrm{~m}$ par an). La sécheresse est aggravée par un vent $\mathrm{sec}$, l'harmattan, qui souffle en saison sèche. Les quelques points d'eau, qui ne tarissent pas, attirent d'importants rassemblements de nomades avec leurs troupeaux. Certaines années, le problème de l'abreuvement est véritablement crucial, sans parler de celui de la famine due aux mauvaises récoltes consécutives à une pluviométrie qui a baissé de moitié durant quatre ans.

Différentes ethnies se rencontrent dans ces régions : nomades, semi-nomades ou sédentaires, mais les populations sont en général à vocation pastorale, tels les Peuls, les Bellas et les Touaregs. Les quelques cultures pratiquées sont céréalières (mil et sorgho surtout). La vie de ces régions est dominée par l'élevage : zébus, chèvres et moutons sont largement représentés, et ânes, chameaux et chevaux sont utilisés pour le transport. Au hasard des déplacements, on rencontre fréquemment d'immenses troupeaux de zébus, avec leurs bergers, à la recherche de quelques touffes d'une herbe desséchée et si rare qu'elle n'en est devenue que plus précieuse en fin de saison sèche.

\subsection{Savane sud-soudanienne ou préguinéenne}

Deux enquêtes ont eu lieu en Haute-Volta (régions de Banfora et de Gaoua). Ce sont des zones à pluviométric moyenne $(1000$ à $1200 \mathrm{~mm}$ par an), avec une saison sèche de 7 mois (octobre à avril) et une saison des pluies de 5 mois (mai à septembre). C'est une savane arborée à hautes herbes avec quelques cours d'eau permanents, mais à débit très irrégulier. Les ethnies y sont nombreuses. Du point de vue agricole, à côté des cultures vivrières traditionnelles destinées principalement à la consommation locale (mil, sorgho, maïs, ignames), la principale culture d'exportation est constituée par le coton. Un important complexe sucrier est en cours d'installation près de Banfora avec de vastes plantations de canne à sucre. Malgré tout, l'élevage est encore important : grands et petits ruminants surtout, mais aussi quelques ânes et porcs. Toutefois, les propriétaires d'animaux ne s'occupent pas eux-mêmes, la plupart du temps, des ruminants, mais en confient la garde et l'entretien à un ou deux bergers peuls.

\subsection{Savane guinéenne ou préforestière}

Trois enquêtes ont eu lieu en Côte d'Ivoire (Bouaké, Katiola, Korhogo et Odienné). Ce sont des régions de savane boisée à pluviométrie plus importante (1 200 à $1600 \mathrm{~mm}$ par an) avec une saison sèche de 4 à 5 mois, et une saison des pluies de 7 à 8 mois (mai à octobre). Les ethnies sont variables selon les régions: Baoulés près de Bouaké, Sénoufos et Dioulas 
Etude épidémiologique de la brucellose en Afrique

Situation géographique des régions prospectées

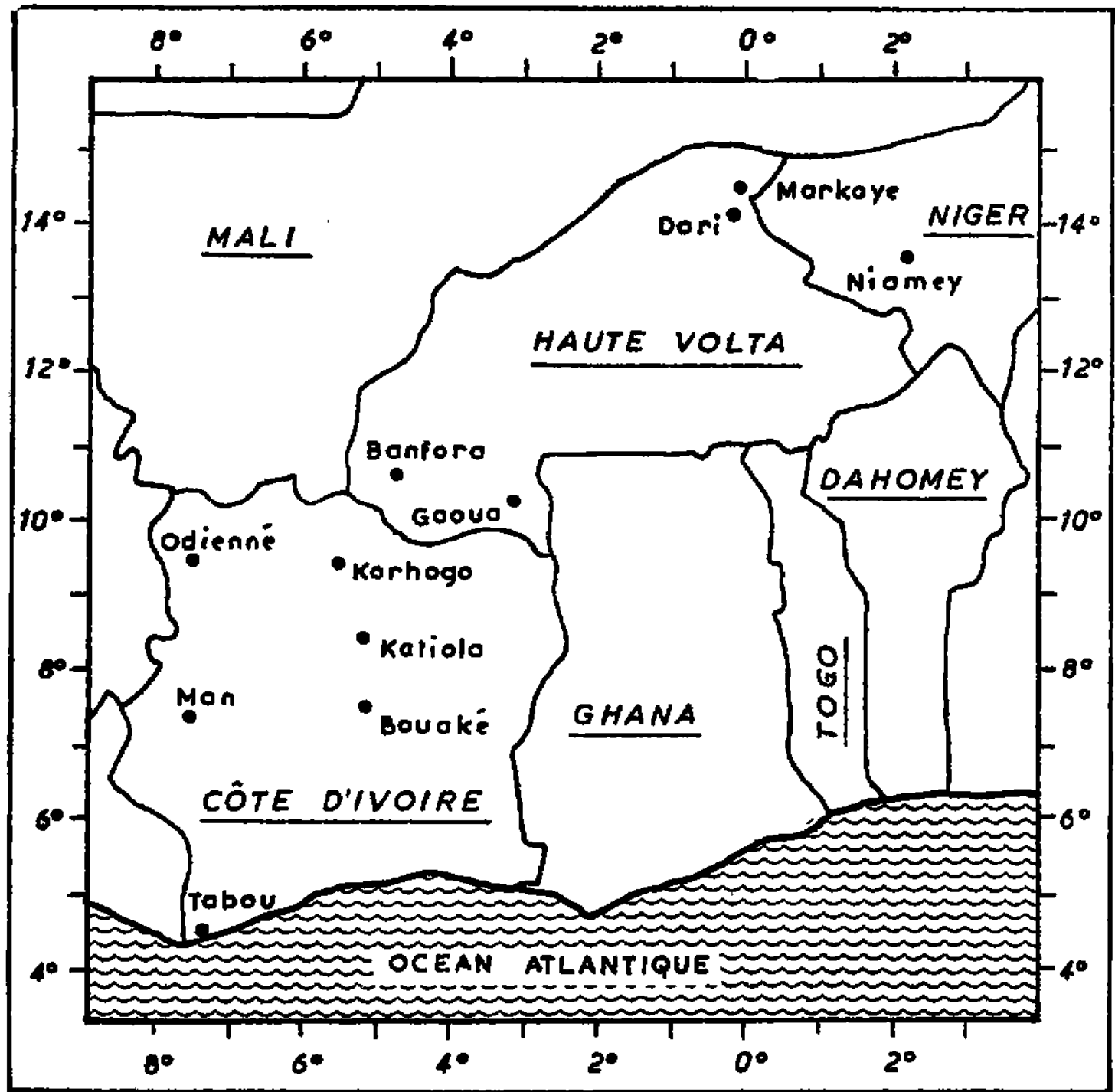

près de Korhogo, Malinkés près d’Odienné. Ces populations pratiquent les cultures vivrières traditionnelles : mil, sorgho, maïs, riz, ignames, et une culture d'exportation: le coton. L'élevage est en développement et relativement important dans le Nord: grands et petits ruminants partout et porcins près de Korhogo.

\subsection{Régions de forêts}

Deux enquêtes ont été effectuées en Côte d'Ivoire (Man et Tabou). Ce sont des régions à pluviométrie relativement élevée $(1750 \mathrm{~mm}$ à Man, $2300 \mathrm{~mm}$ à Tabou). Man, au relief fortement accidenté, est une zone de transition entre la forêt dense au Sud et la savane guinéenne au Nord. Les ressources agricoles sont variées : cultures vivrières, bananes, café, cacao, palmiers à huile, exploitation du bois. En matière d'élevage, on rencontre grands et petits ruminants, mais ces derniers prédominent, en particulier les ovins.

Tabou est une région de forêt dense, située en bordure de mer, peu peuplée, sous-exploitée actuellement mais qui va faire l'objet d'un vaste plan de mise en valeur (plantations de palmiers à huile). L'élevage est presque inexistant, à part quelques chèvres et moutons et quelques très rares bovins.

$\mathrm{Du}$ point de vue ethnies, on rencontre les Dan et Wobé à Man et les Kroos et Yacoubas à Tabou. 


\section{METHODOLOGIE DES ENQUETES}

\subsection{Sur le terrain}

Dans chacune des régions étudiées, une douzaine de villages en moyenne ont été prospectés (8 à 17, selon les cas, en éliminant les villages inaccessibles).

L'équipe chargée de ces enquêtes comprenait 1 ou 2 médecins, 1 vétérinaire, 1 technicien de laboratoire, infirmiers, mancuvres et chauffeurs. Elle disposait d'un camion pour le transport du gros matériel (réfrigérateurs, congélateurs, groupes électrogènes, bouteilles à gaz, matériel de camping, etc.) et de 3 ou 4 LandRover, selon les enquêtes.

Chaque jour 1 ou 2 villages étaient prospectés. Ceux-ci, déjà prévenus par l'administration générale (préfets, sous-préfets ou commandants de cercle) selon un programme établi par avance, étaient informés à nouveau la veille de la prospection par les soins de notre équipe accompagnée d'un agent du service de l'Elevage et du service de Santé. Cette précaution s'avère indispensable si on veut avoir un taux de présence suffisant, que la population se présente à jeun et que les animaux soient gardés au village (et non pas lâchés en brousse) et les femelles laitières non traites (afin de pouvoir effectuer les ring-tests sur le lait).

Un laboratoire de campagne était installé en base fixe au centre de la région prospectée. C'est là qu'étaient entreposés réfrigérateurs et congélateurs à gaz, centrifugeuses fonctionnant en général sur groupes électrogènes, stérilisateurs, autoclaves, etc.

\subsubsection{Chez les animaux}

La priorité a été donnée aux recherches chez les bovins. Toutefois, et dans la mesure des possibilités matérielles, les recherches ont intéressé également les petits ruminants (et surtout les caprins) en particulier dans les régions de grand élevage, et, très accessoirement, les ânes.

Dans chaque village prospecté, toutes les femelles laitières présentes, ainsi que les chèvres et brebis en lactation dont nous avons pu obtenir la présentation, ont été l'objet d'un ring-test individuel.

Les ring-tests ont été effectués sur le terrain suivant la technique classique (RENOUX G. et GAUMONT R., 1966). Nous avons utilisé l'antigène brucellique coloré $\mathrm{BD}$ Mérieux qui était stocké en réfrigérateur et transporté sur le terrain en boîtes à glace. La réaction était exécutée en tubes à hémolyse stériles. Les lectures étaient faites sur place le plus souvent pour les laits de bovins. Par contre, les ringtests effectués sur les laits de petits ruminants demandant un temps de repos de $6 \mathrm{~h}$ au minimum après adjonction de l'antigène, les tubes étaient rapportés à notre base, et la lecture effectuée dans la soirée. Les résultats étaient classés en positifs, douteux et négatifs.

Dans la majorité des villages, en particulier lorsque le nombre des ring-tests n'était pas très élevé, des prises de sang ont été effectuées chez les bovins principalement, mais aussi chez quelques caprins et ovins, et parfois quelques ânes. Les tubes de sang étaient transportés en boîtes à glace à notre base. Après centrifugation, les sérums recueillis étaient placés en tubes stériles (tubes en plastique) puis conservés en congélateurs.

Dans les régions sahéliennes, par suite des conditions d'élevage et de l'absence de parcs à animaux rendant la contention de ceux-ci aléatoire, les prises de sang s'avéraient difficiles à effectuer et demandaient surtout beaucoup de temps. Aussi avons-nous dû nous limiter la plupart du temps aux seuls ring-tests. Il est vrai que les femelles laitières étaient en général suffisamment nombreuses dans ces régions.

Les commémoratifs éventuels concernant les avortements ou la détection d'hygromas dans les troupeaux étaient notés.

\subsubsection{Chez lhomme}

Dans les villages visités, selon l'importance numérique de la population, l'enquête a été soit exhaustive, soit réalisée par sondage aléatoire, après stratification préalable de la population présente en 4 groupes, garçons et filles âgés de 1 à 14 ans, et hommes et femmes âgés de 15 ans et plus.

Les sujets retenus se présentaient en premier lieu au secrétariat où leur nom, leur âge et leur sexe étaient notés. Un numéro leur était attribué et il leur était remis un papier portant ce numéro, ainsi que leur nom et prénom, (il arrive en effet que certains sujets échangent 
leurs papiers, ou encore que le chef de famille ramasse tous les papiers de sa famille qu'il redistribuera plus tard selon sa fantaisie).

Les personnes étaient ensuite soumises à une double intradermoréaction (mélitine d une part et témoin d'autre part). L'antigène à la mélitine et le témoin que nous avons utilisés nous ont été fournis par l'Institut Pasteur de Paris. La lecture de la réaction était effectuée le lendemain par rapport au témoin. Les réactions égales ou supérieures à $5 \mathrm{~mm}$, avec un témoin rigoureusement négatif, ont été considérées comme positives (J. ROUX, communication personnelle).

Aussitôt après les 2 intradermoréactions, une prise de sang de $10 \mathrm{ml}$ était effectuée chez chaque sujet. Les sangs recueillis étaient traités et conservés comme indiqué précédemment.

\subsection{Au laboratoire à Bobo-Dioulasso}

Les sérums humains ont été en premier lieu l'objet d'une séro-agglutination de Wright. Puis tous ceux ayant présenté une réaction positive ou douteuse, même très faible, ont été testés en déviation du complément.

Les sérums animaux ont été testés systématiquement à la fois en séro-agglutination et en fixation du complément.

\subsubsection{Séro-agglutination de Wright}

L'antigène utilisé a été celui de BD-Mérieux. standardisé par rapport à l'étalon international de sérum anti-Brucella abortus (agglutination ++ avec le sérum international dilué au $1 / 650$ ).

La réaction a été effectuée sur six tubes, afin de prévenir un phénomène de zone éventuel (dilutions du 1/10 au 1/320). La lecture a été effectuée après 24 heures d'étuve à $37^{\circ}$ en se basant, non pas sur l'importance de l'agglutination, mais sur la densité optique du liquide surnageant $(1,5)$. La plus forte dilution de sérum où l'on observe au moins $50 \mathrm{p}$. 100 d'agglutination (c'est-à-dire 50 p. 100 de clarification) est considérée comme le point 50 p. 100 ou titre du sérum étudié. En raison de l'importance particulière de ce point $50 \mathrm{p}$. 100 on prépare un tube témoin simulant 50 p. 100 de clarification en mélangeant, dans un tube à agglutination (tube à hémolyse),
$0,25 \mathrm{ml}$ d'antigène et $0,75 \mathrm{ml}$ d'eau salée. La lecture des agglutinations est effectuée en comparant l'opacité du surnageant des six tubes de la réaction (que l'on prend soin de ne pas secouer) à l'opacité du surnageant du tube témoin qui correspond, en opacité, à ++ . L'échelle de notation adoptée a été la suivante (1, loc. cit.) :

$$
\begin{aligned}
++++= & \begin{array}{c}
\text { Agglutination complète et clari- } \\
\text { fication complète. }
\end{array} \\
+++= & \begin{array}{l}
\text { Agglutination à peu près com- } \\
\text { plète et } 75 \text { p. } 100 \text { de clarification } \\
\text { du surnageant. }
\end{array} \\
++= & \begin{array}{l}
\text { Agglutination marquée et clari- } \\
\text { fication égale à } 50 \text { p. } 100 .
\end{array} \\
+= & \begin{array}{l}
\text { Légère agglutination et } 25 \text { p. } 100 \\
\text { de clarification. }
\end{array} \\
-= & \text { Absence de toute agglutination } \\
& \text { et de toute clarification. }
\end{aligned}
$$

Suivant les recommandations de l'OMS, la teneur en agglutinines a été exprimée en Unités Internationales et n'ont été considérés comme positifs que les sérums titrant au moins 100 U.I. par ml pour les sérums humains et bovins, et plus de 50 U.I. par $\mathrm{ml}$ pour les sérums de petits ruminants (4).

En fait, étant donné les normes de l'antigène que nous avons utilisé et les dilutions pratiquées, nous n'avons retenu comme positifs que les sérums titrant au moins 123 U.I. chez les humains et bovins et 62 U.I. chez les petits ruminants.

\subsubsection{Fixation du complément}

La technique que nous avons employée est celle du type Kolmer avec fixation à froid du complément. C'est celle qui est la plus répandue $(1$, loc. cit.; 5 , loc. cit.). Comme liquide de dilution nous avons d'abord utilisé un tampon au véronal ou tampon de Mayer. Puis, par suite de l'impossibilité d'obtenir la préparation d'un tampon au véronal correct, nous avons eu recours à la technique préconisée par RENOUX et GAUMONT (loc. cit.) qui fait appel, comme liquide de dilution, à de l'eau physiologique à 0,85 p. 100 additionnée de $1 \mathrm{ml}$ par litre de sulfate de magnésium $7 \mathrm{H} 2 \mathrm{O}$ à 10 p. 100. Pour l'exécution pratique, nous nous sommes conformés aux indications données par les mêmes auteurs. Les sérums ont notamment été inactivés par chauffage de 
30 minutes à $60^{\circ}$, ce qui a permis de réduire le nombre de sérums anticomplémentaires, fréquents en Afrique chez l'homme (J. ROUX, communication personnelle).

La réaction a été effectuée avec 4 tubes (dilutions du $1 / 4$ au 1/32). Les résultats ont été notés de : - (hémolyse totale) à ++++ (absence complète d'hémolyse).

\section{RESULTATS CHEZ LES ANIMAUX}

Les résultats figurent au tableau I en ce qui concerne les ring-tests et au tableau II pour les examens sérologiques.

Les sérums, tant humains qu'animaux, ont été notés comme positifs lorsque l'un au moins des 2 tests pratiqués (séro-agglutination de Wright et fixation du complément) est apparu positif (voir précédemment les critères de positivité) et ils ont été classés comme douteux lorsque la séro-agglutination et la fixation du complément ont été douteuses ou lorsque la séro-agglutination seule a été douteuse (avec un titre de 62 U.I. au moins, le sérum étant anticomplémentaire.

\subsection{Chez les bovins}

La maladie est rencontrée dans toutes les régions prospectées à un degré plus ou moins important, sauf dans les régions sahéliennes de Haute-Volta où elle reste à un niveau très modéré. Dans ces dernières régions, certains villages sont même apparus entièrement indemnes, tels ceux de Selbo (région de Dori) où les 64 ring-tests ont été totalement négatifs, et de Darkoy (région de Markoy) où les 46 ringtests ont été tous négatifs. D'autres villages sont très peu infectés, tel celui de Diomga (région de Dori) avec un seul ring-test positif sur 55 .

L'endémie semble de plus en plus importante au fur et à mesure que l'on descend

- TABLEAU N ${ }^{\circ}$. - Résultats des Ring-test chez les animaux.

\begin{tabular}{|c|c|c|c|c|c|c|c|c|c|}
\hline \multirow{2}{*}{$\begin{array}{c}\text { Régions } \\
\text { prospectées }\end{array}$} & \multicolumn{3}{|c|}{$B \subset \forall i n s$} & \multicolumn{3}{|c|}{$C$ a $p$ r ins } & \multicolumn{3}{|c|}{$0 \mathrm{v} i \mathrm{~ns}$} \\
\hline & $\mathrm{T}$ & $\begin{array}{c}+ \\
\text { Nombre et } \\
\text { p.100 }\end{array}$ & $\begin{array}{c}\stackrel{+}{\text { Nombre et }} \\
\text { p. } 100\end{array}$ & $\mathrm{~T}$ & $\begin{array}{c}+ \\
\text { Nombre et } \\
\text { p. } 100\end{array}$ & $\begin{array}{c}+ \\
\text { Nombre et } \\
\text { p. } 100\end{array}$ & $\mathbf{T}$ & $\begin{array}{c}+ \\
\text { Nombre et } \\
\text { p.100 }\end{array}$ & $\begin{array}{c}+ \\
\text { Nombre et } \\
\text { p.100 }\end{array}$ \\
\hline $\begin{array}{l}\text { Bouaké } \\
\text { (Côte d'Ivoire) }\end{array}$ & 700 & $\begin{array}{l}359 \\
(51,0)\end{array}$ & $\begin{array}{l}185 \\
(26,4)\end{array}$ & 4 & 1 & - & 14 & 4 & 4 \\
\hline $\begin{array}{l}\text { Korhogo } \\
\text { (côte d'Ivoire) }\end{array}$ & 335 & $\begin{array}{l}130 \\
(38,8)\end{array}$ & $\begin{array}{c}90 \\
(26,9)\end{array}$ & 15 & 5 & 1 & 4 & 3 & - \\
\hline $\begin{array}{l}\text { Man } \\
\text { (Côte d'Ivoire) }\end{array}$ & 100 & $\begin{array}{c}23 \\
(23,0)\end{array}$ & $(5,0)$ & 11 & 5 & - & 15 & 2 & 4 \\
\hline $\begin{array}{l}\text { Odienne } \\
\text { (Côte d'Ivoire) }\end{array}$ & 192 & $\begin{array}{c}57 \\
(29,7)\end{array}$ & $\begin{array}{l}18 \\
(9,4)\end{array}$ & $B$ & 1 & - & 39 & $\begin{array}{l}4 \\
(10,3)\end{array}$ & 1 \\
\hline $\begin{array}{l}\text { Tabou } \\
\text { (Côte d'Ivoire) }\end{array}$ & - & - & - & - & - & - & - & - & - \\
\hline $\begin{array}{l}\text { Banfora } \\
\text { (Haute-Volta) }\end{array}$ & 270 & $\begin{array}{c}58 \\
(21,5)\end{array}$ & $\begin{array}{l}14 \\
5,2)\end{array}$ & 121 & $\begin{array}{c}20 \\
(16,8)\end{array}$ & $\begin{array}{c}13 \\
(10,7)\end{array}$ & 116 & $\begin{array}{c}13 \\
(11,2)\end{array}$ & $\left(\begin{array}{l}4 \\
3,4)\end{array}\right.$ \\
\hline $\begin{array}{l}\text { Dori } \\
\text { (Haute-Volta) }\end{array}$ & 499 & $\begin{array}{l}30 \\
(6,0)\end{array}$ & $\begin{array}{l}12 \\
2,4)\end{array}$ & 251 & $\begin{array}{l}12 \\
4,8)\end{array}$ & $\begin{array}{c}8 \\
(3,2)\end{array}$ & - & - & - \\
\hline $\begin{array}{l}\text { Gaoua } \\
\text { (Haute-Vo1ta) }\end{array}$ & 67 & $\begin{array}{c}21 \\
(31,3)\end{array}$ & - & 7 & 2 & - & - & - & - \\
\hline $\begin{array}{l}\text { Markoye } \\
\text { (Haute-Volta) }\end{array}$ & 499 & $\begin{array}{l}44 \\
(8,8)\end{array}$ & $\begin{array}{l}21 \\
(4,2)\end{array}$ & 411 & $\begin{array}{c}48 \\
(11,7)\end{array}$ & $\begin{array}{l}12 \\
2,9)\end{array}$ & - & - & - \\
\hline $\begin{array}{l}\text { Niamey } \\
\text { (Niger) }\end{array}$ & 245 & $\begin{array}{c}52 \\
(21,2)\end{array}$ & $\begin{array}{c}63 \\
(25,7)\end{array}$ & 104 & $\begin{array}{c}47 \\
(45,1)\end{array}$ & $\begin{array}{c}27 \\
(26,0)\end{array}$ & 81 & $\begin{array}{c}18 \\
(22,2)\end{array}$ & $\begin{array}{c}24 \\
(29,6)\end{array}$ \\
\hline Total & 2907 & $\begin{array}{c}774 \\
(26,6)\end{array}$ & $\begin{array}{c}408 \\
(14,0)\end{array}$ & 932 & $\begin{array}{l}141 \\
(15,1)\end{array}$ & $\begin{array}{l}61 \\
(6,5)\end{array}$ & 269 & $\begin{array}{c}44 \\
(16,4)\end{array}$ & $\begin{array}{c}37 \\
(13,8)\end{array}$ \\
\hline
\end{tabular}

Rectification: in 3e colonne de "Bovins", "Caprins", "Ovins": lire \pm au lieu de + de même que pour le tableau $\mathrm{n}^{\circ}$ II. 
TABLEAU N ${ }^{\circ}$ II. - Résulcats sérologiques chez les animaux.

\begin{tabular}{|c|c|c|c|c|c|c|c|c|c|}
\hline \multirow{2}{*}{$\begin{array}{c}\text { Régions } \\
\text { prospectëes }\end{array}$} & \multicolumn{3}{|c|}{$B \circ v i n s$} & \multicolumn{3}{|c|}{$\mathrm{Cap}$ a ins } & \multicolumn{3}{|c|}{$0 v i n s$} \\
\hline & T & $\begin{array}{c}+ \\
\text { Nombre et } \\
\text { p. } 100\end{array}$ & $\begin{array}{c}+ \\
\text { Nombre et } \\
\text { p. } 100\end{array}$ & $\mathrm{~T}$ & $\begin{array}{c}+ \\
\text { Nombre et } \\
\text { p. } 100\end{array}$ & $\begin{array}{c}+ \\
\text { Nombre et } \\
\text { p. } 100\end{array}$ & $\mathrm{~T}$ & $\begin{array}{c}\stackrel{+}{\text { Nombre et }} \\
\text { p.100 }\end{array}$ & $\begin{array}{c}+ \\
\text { Nombre et } \\
\text { p. } 100\end{array}$ \\
\hline $\begin{array}{l}\text { Bouaké } \\
\text { (Côte d'Ivoire) }\end{array}$ & 114 & $\left(\begin{array}{c}3 \\
2,6)\end{array}\right.$ & $\begin{array}{l}11 \\
(9,6)\end{array}$ & 2 & - & - & - & - & - \\
\hline $\begin{array}{l}\text { Korhogo } \\
\text { (Côte d'Ivoire) }\end{array}$ & 347 & $\begin{array}{c}54 \\
(15,6)\end{array}$ & $\begin{array}{c}8 \\
(2,3)\end{array}$ & 30 & - & - & 6 & - & - \\
\hline $\begin{array}{l}\text { Man } \\
\text { (Côte d'Ivoire) }\end{array}$ & 125 & $\begin{array}{c}17 \\
(13,6)\end{array}$ & - & 2 & - & - & 2 & - & - \\
\hline $\begin{array}{l}\text { Odienne } \\
\text { (Côte d'Ivoire) }\end{array}$ & 163 & $\begin{array}{c}42 \\
(25,8)\end{array}$ & - & 8 & - & - & 4 & - & - \\
\hline $\begin{array}{l}\text { Tabou } \\
\text { (Côte d'Ivoire) }\end{array}$ & - & - & - & 6 & - & - & 7 & - & - \\
\hline $\begin{array}{l}\text { Banfora } \\
\text { (Haute-Volta }\end{array}$ & 183 & $\begin{array}{c}20 \\
(10,9)\end{array}$ & - & 45 & - & - & 23 & 1 & - \\
\hline $\begin{array}{l}\text { Dori } \\
\text { (Haute-Wb1ta) }\end{array}$ & - & - & - & - & - & - & - & - & - \\
\hline $\begin{array}{l}\text { Gaoua } \\
\text { (Haute-Volta) }\end{array}$ & 88 & $\left(\begin{array}{c}2 \\
2,3)\end{array}\right.$ & - & 20 & - & - & 6 & - & - \\
\hline $\begin{array}{l}\text { Markoy } \\
\text { (Haute-Volta) }\end{array}$ & - & - & - & - & - & - & - & - & - \\
\hline $\begin{array}{l}\text { Niamey } \\
\text { (Niger) }\end{array}$ & 42 & $\left(\begin{array}{c}1 \\
2,4)\end{array}\right.$ & - & - & - & - & - & - & - \\
\hline Total (1) & 1062 & $\begin{array}{l}139 \\
(13,1)\end{array}$ & $(1,8)$ & 101 & $\begin{array}{c}0 \\
(0,0)\end{array}$ & $\begin{array}{c}0 \\
(0,0)\end{array}$ & 42 & $\begin{array}{c}1 \\
(2,4)\end{array}$ & - \\
\hline
\end{tabular}

(1) Il y a lieu d'ajouter au total général les 20 examens sérologıques effectués chez les ânes.

vers le Sud (sauf la région de Tabou, où l'élevage est pratiquement inexistant). C'est ainsi que l'on trouve seulement 6 p. 100 de ring-tests positifs dans la région de Dori et 51 p. 100 dans celle de Bouaké. Dans cette dernière, ainsi que dans la région de Korhogo, certains villages sont apparus très infectés, tels celui de Yékolo (Bouaké) avec 70 p. 100 de ring-tests positifs, ceux de Blaouara et Tiototiéri près de Korhogo avec respectivement 100 p. 100 et 75 p. 100 , celui de Lakpolo (Korhogo) avec 63 p. 100 de sérologies positives.

Dans ces régions, où des moyens considérables sont mis en œuvre pour le développement de l'élevage, il s'avère nécessaire d'envisager dès maintenant des mesures de prophylaxie médicale et sanitaire, sous peine de voir les efforts, entrepris pour l'introduction et la promotion de l'élevage dans les populations rurales, dangereusement compromis, alors que par ailleurs de nombreuses conditions favorables semblent réunies.
Il convient de noter une certaine discordance entre les résultats sérologiques et ceux des ring-tests. Il ne s'agit pas, bien sûr, des mêmes animaux, et ceux qui ont fait l'objet d'un prélèvement de sang sont, souvent, de jeunes animaux. Ces faits ont été en particulier observés dans la région de Bouaké où pour 51 p. 100 de ring-tests positifs, nous n'avons eu que 2,6 p. 100 de sérums positifs, avec, il est vrai, 9,6 p. 100 de sérums douteux. Par contre, à Odienné par exemple, pour 29,7 p. 100 de ring-tests positifs, nous avons eu 25,8 p. 100 de sérologies positives. Nous n'avons pas trouvé d'explication réellement satisfaisante à ces discordances. Précisons que pour les ring-tests, il ne s'agit pas de fausses réactions positives, car dans tous les troupeaux fortement infectés, des avortements nous ont été signalés par les éleveurs et confirmés par le service de l'Elevage, et nous avons pu observer nous-mêmes d'assez nombreux cas d'hygromas. L'étiologie brucellique ne fait donc pas de doute. 


\subsection{Chez les petits ruminants}

L'endémie apparaît globalement moins importante que chez les bovins. Cependant certains chiffres portent sur des effectifs trop faibles (notamment ceux concemant les enquêtes effectuées en Côte d'Ivoire) pour qu'ils soient interprétables statistiquement. A noter cependant que les 101 sérologies effectuées chez les caprins ont été toutes négatives et qu'un seul examen sérologique sur 42 a été positif chez les ovins.

\subsection{Chez les ânes}

Vingt examens sérologiques seulement ont été pratiqués : 5 dans la région de Banfora avec 2 résultats positifs et 15 dans celle de Niamey avec un positif. Ces résultats montrent néanmoins que ces animaux peuvent être réservoirs de virus.

\section{INCIDENCE DE LA BRUCELLOSE CHEZ L'HOMME}

\subsection{Résultats globaux}

Les résultats d'ensemble figurent au tableau III.

12739 intradermoréactions à la mélitine ont été pratiquées au total, mais seulement 10830 ont pu être contrôlées par suite d'un absentéisme variable selon les régions et les villages. 11990 examens sérologiques ont été effectués (chez certains sujets, notamment les très jeunes enfants, la prise de sang n'a pu être réalisée).

Dans les régions de savane soudanienne et guinéenne et les zones forestières, peuplées d'agriculteurs souvent propriétaires d'animaux, mais ne s'occupant pas habituellement euxmêmes de ceux-ci, l'incidence de la maladie est faible en général, sauf chez les bergers et leurs familles.

TABLEAU $N^{\bullet} I I I .-$ Résultats allergologzques et sérologiques chez $1^{\prime}$ homne

\begin{tabular}{|c|c|c|c|c|c|}
\hline \multirow{2}{*}{$\begin{array}{c}\text { Régions } \\
\text { prospectées }\end{array}$} & \multicolumn{2}{|c|}{$\begin{array}{l}\text { I D R } \\
\text { Mêlitine }\end{array}$} & \multicolumn{3}{|c|}{ Examens sêrologiques } \\
\hline & $\mathrm{T}$ & $\begin{array}{c}+ \\
\text { Nombre et } \\
\text { p.100 }\end{array}$ & $\mathrm{T}$ & $\begin{array}{c}\stackrel{+}{\text { Nombre et }} \\
\text { p. } 100\end{array}$ & $\begin{array}{c}+ \\
\text { Nombre et } \\
\text { p.100 }\end{array}$ \\
\hline $\begin{array}{l}\text { Bouaké } \\
\text { (Côte d'Ivoire) }\end{array}$ & 1025 & $\left(\begin{array}{c}73 \\
(7,1)\end{array}\right.$ & 1122 & $\left(\begin{array}{l}11 \\
(1,0)\end{array}\right.$ & $\begin{array}{l}10 \\
(0,9)\end{array}$ \\
\hline $\begin{array}{l}\text { Korhogo } \\
\text { (Côte d'Ivoire) }\end{array}$ & 1557 & $\begin{array}{l}133 \\
(8,5)\end{array}$ & 1629 & $\left(\begin{array}{c}6 \\
0,4)\end{array}\right.$ & $\left(\begin{array}{c}6 \\
0.4)\end{array}\right.$ \\
\hline $\begin{array}{l}\text { Man } \\
\text { (Côte d'Ivoire) }\end{array}$ & 789 & $\begin{array}{c}49 \\
(6,2)\end{array}$ & 780 & $\left(\begin{array}{c}6 \\
0,8)\end{array}\right.$ & $\begin{array}{l}10 \\
(1,3)\end{array}$ \\
\hline $\begin{array}{l}\text { Odienne } \\
\text { (Côte d'Ivoire) }\end{array}$ & 938 & $\left(\begin{array}{c}67 \\
7,1)\end{array}\right.$ & 970 & $\left(\begin{array}{c}2 \\
0,2)\end{array}\right.$ & $\left(\begin{array}{c}8 \\
0, B)\end{array}\right.$ \\
\hline $\begin{array}{l}\text { Tabou } \\
\text { (Côte d'Ivoire) }\end{array}$ & 1021 & $\left(\begin{array}{c}26 \\
(2,5)\end{array}\right.$ & 992 & $\left(\begin{array}{c}0 \\
0,0)\end{array}\right.$ & $\left(\begin{array}{c}6 \\
0.6\end{array}\right)$ \\
\hline $\begin{array}{l}\text { Banfora } \\
\text { (Haute-Volta) }\end{array}$ & 1268 & $\begin{array}{c}78 \\
(6,2)\end{array}$ & 1357 & $\left(\begin{array}{c}5 \\
0,4\end{array}\right)$ & $\begin{array}{c}7 \\
0,5)\end{array}$ \\
\hline $\begin{array}{l}\text { Dori } \\
\text { (Haute-Volta) }\end{array}$ & 838 & $\begin{array}{c}252 \\
(30,1)\end{array}$ & 985 & $\begin{array}{c}99 \\
(10,1)\end{array}$ & $\begin{array}{l}18 \\
(1,8)\end{array}$ \\
\hline $\begin{array}{l}\text { Gaoua } \\
\text { (Haute-Volta) }\end{array}$ & 1040 & $\left(\begin{array}{c}24 \\
2,3)\end{array}\right.$ & 1622 & $\left(\begin{array}{c}3 \\
0,2\end{array}\right)$ & $\left(\begin{array}{c}1 \\
0,1)\end{array}\right.$ \\
\hline $\begin{array}{l}\text { Markay } \\
\text { (Haute-Volta) }\end{array}$ & 1273 & $\begin{array}{c}173 \\
(13,6)\end{array}$ & 1340 & $\left(\begin{array}{l}27 \\
(2,0)\end{array}\right.$ & $\begin{array}{l}19 \\
(1,4)\end{array}$ \\
\hline $\begin{array}{l}\text { Niamey } \\
\text { (Niger) }\end{array}$ & 1081 & $\begin{array}{c}66 \\
(6,1)\end{array}$ & 1193 & $\begin{array}{l}17 \\
(1,4)\end{array}$ & $\left(\begin{array}{c}6 \\
0,5)\end{array}\right.$ \\
\hline Total & 10830 & $\begin{array}{l}941 \\
(8,7)\end{array}$ & 11990 & $\begin{array}{l}176 \\
(1,5)\end{array}$ & $\begin{array}{l}91 \\
(0,8)\end{array}$ \\
\hline
\end{tabular}

Rectification : in $2^{e}$ colonne \& IDR Mélitine » et in $3^{e}$ colonne « Examens sérologiques » : lire \pm au lieu de + . 
La positivité des intradermoréactions va de 2,5 p. 100 (Tabou en Côte d'Tvoire) à 8,5 p. 100 (Korhogo en Côte d'Ivoire) et celle des sérologies de 0 p. 100 (Tabou) à 1 p. 100 (Bouaké en Côte d'Ivoire). Cependant, on peut observer que certains villages sont nettement plus infectés que ces chiffres moyens. Il en a été ainsi de Kpenguérékaha (près de Korhogo) avec 17 p. 100 d'intradermoréactions positives, Sokorodougou près d'Odienné (Côte d'Ivoire) avec 15 p. 100 , Sindou avec 16 p. 100 et Sidéradougou avec 13 p. 100 , tous deux situés dans la région de Banfora en Haute-Volta. Les renseignements recueillis auprès des habitants de ces villages nous ont appris que certaines familles avaient coutume de consommer régulièrement du lait.

Par contre dans les régions sahéliennes prospectées, c'est là que furent observés les plus forts taux d'infection dans la population humaine.

Il convient de mettre à part les résultats obtenus au Niger où une seule enquête a été réalisée dans la région de Niamey. En effet, les villages visités, trop proches de la capitale, étaient essentiellement peuplés de citadins, résidant dans ces villages, mais travaillant à la ville. De ce fait, les résultats ne reflètent pas l'incidence réelle de l'affection chez l'homme, dans les populations rurales, à vocation pastorale qui constituent en fait la majorité de la population du Niger.

Dans les régions sahéliennes de Haute-Volta, malgré la faible endémie animale (6 à 8 p. 100 de ring-tests positifs chez les bovins, 4 à $11 \mathrm{p}$. 100 chez les caprins) des taux élevés d'infection ont été observés chez l'homme : 30 p. 100 d'intradermoréactions positives à Dori, $14 \mathrm{p}$. 100 à Markoy, et 10 p. 100 de sérologies positives à Dori. Dans cette dernière région, plusieurs villages ont montré près de 20 p. 100 ou plus de sérologies positives et l'un d'entre eux plus de 50 p. 100 d'intradermoréactions positives.

Ces faits qui, de prime abord, peuvent sembler paradoxaux s'expliquent, en fait, fort bien et montrent le rôle majeur des conditions écologiques dans la transmission de la brucellose à l'homme. Il faut noter aussi que des variations importantes (dont nous reparlerons plus loin) sont observées en fonction des ethnies, ceci étant lié au mode de vie des populations, aux élevages que celles-ci pratiquent (grands ou petits ruminants), à la consommation ou non de laitages.

\subsection{Variations des résultats allergologiques en fonction de l'âge et du sexe (voir tableau IV)}

Les adultes âgés de 15 ans et plus apparaissent globalement très significativement plus positifs que les enfants $(10,8$ p. 100 pour 3,7 p. 100). Ces résultats ne sont pas pour surprendre car l'intradermoréaction, restant positive plusieurs années chez un sujet, traduit non seulement l'état immunitaire présent mais également passé de cet individu, et il est évident qu'un adulte aura eu plus d'occasions de contagion qu'un enfant. Toutefois, étudiées par village, les différences observées ne sont pas systématiquement en faveur des adultes, et on rencontre de temps à autre un village où la différence est en faveur des enfants, ceci étant lié au mode de vie des populations : par exemple, enfants chargés de la garde des troupeaux dans certains villages.

Une positivité globalement très significative apparaît également au profit des sujets de sexe masculin (12,0 p. 100 pour 5,2 p. 100$)$. Cette différence entre les sexes est particulièrement nette et significative chez les adultes (hommes : 15,3 p. 100 ; femmes: 6,2 p. 100 ). Par contre, si une différence existe globalement entre les garçons et les filles au profit des premiers (4,4 p. 100 pour 2,7 p. 100 ) celle-ci, tout en étant significative, est peu marquée et n'est retrouvée dans aucune enquête au niveau des régions.

Les résultats en fonction du sexe et de l'âge s'expliquent là encore fort bien. Les possibilités de contagion pour les jeunes enfants sont les mêmes, quelque soit le sexe. Chez les populations pastorales du nord, ce n'est qu'à partir de l'âge de 7 ou 8 ans que les garçons commencent à être initiés à la garde des troupeaux, tandis que les fillettes ont la charge des veaux qui restent au village. Mais par la suite, et dans la plupart des régions, les occasions de contact deviendront plus fréquentes pour les hommes. Mais ici aussi, on peut observer, en particulier dans certains villages du sahel, un taux d'infection supérieur chez les femmes. Ceci tient aux coutumes de certaines ethnies où ce sont tradi- 


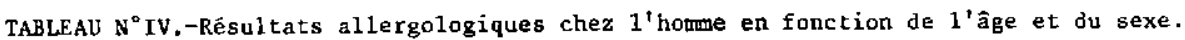

\begin{tabular}{|c|c|c|c|c|}
\hline \multicolumn{2}{|c|}{ Facteurs étudiês } & \multirow{2}{*}{$\begin{array}{c}\begin{array}{c}\text { Nombre de } \\
\text { sujets testës }\end{array} \\
3150\end{array}$} & \multirow{2}{*}{$\begin{array}{c}\begin{array}{c}\text { Nombre et } \\
\text { p.100 de } \\
\text { sujets positifs }\end{array} \\
\begin{array}{c}115 \\
(3,7) \\
826 \\
(10,8)\end{array}\end{array}$} & \multirow{2}{*}{$\begin{array}{l}\quad \text { observations } \\
\mathrm{Chi}^{2}=108,13 \\
\text { Lrès significatif } \\
\mathrm{R}<0,000001\end{array}$} \\
\hline Age & $\begin{array}{c}\text { Enfants } \\
(1 \text { a } 14 \text { ans }) \\
\text { Adultes } \\
(15 \text { ans et }+)\end{array}$ & & & \\
\hline Sexe & $\begin{array}{l}\text { Masculin } \\
\text { Féminir }\end{array}$ & $\begin{array}{r}5539 \\
. \quad 5291\end{array}$ & $\begin{array}{c}664 \\
(12,0) \\
\\
277 \\
(5,2)\end{array}$ & $\begin{array}{l}\mathrm{Chi}^{2}=155,5227 \\
\text { très significatif } \\
\mathrm{R}<0,000001\end{array}$ \\
\hline $\begin{array}{l}\text { Age } \\
\text { et } \\
\text { Sexe }\end{array}$ & $\begin{array}{l}\text { Hommes } \\
\text { adultes } \\
\text { Feruoes } \\
\text { adultes }\end{array}$ & $\begin{array}{l}3851 \\
3829\end{array}$ & $\begin{array}{c}589 \\
(15,3) \\
\\
237 \\
(6,2)\end{array}$ & $\begin{array}{l}\text { Chi }^{2}=163,8375 \\
\text { très significatif } \\
R<0,000001\end{array}$ \\
\hline $\begin{array}{l}\text { Age } \\
\text { et } \\
\text { Sexe }\end{array}$ & $\begin{array}{l}\text { Garçons } \\
\text { (1 à } 14 \text { ans) } \\
\text { Filles } \\
\text { (1 à } 14 \text { ans) }\end{array}$ & 1462 & $\begin{array}{c}75 \\
(4,4) \\
40 \\
(2,7)\end{array}$ & $\begin{array}{l}\mathrm{Chi}^{2}=6,4 \mathrm{B66} \\
\text { significatif } \\
\mathrm{R}<0,02\end{array}$ \\
\hline
\end{tabular}

tionnellement les femmes qui ont la responsabilité de la traite du lait : il en est ainsi chez les Dielgobés et les Gaobés par exemple.

\subsection{Résultats chez les bergers et les non-bergers (voir tableau $\mathrm{V}$ )}

Il est de tradition que les agriculteurs, lorsqu'ils sont propriétaires d'animaux, ne s'occupent pas en général eux-mêmes de ceux-ci, mais en confient la garde et l'entretien à des bergers peuls qui sont rémunérés en nature par le lait qu'ils prélèvent. Cette coutume est regrettable, car elle présente de nombreux inconvénients pour le développement de l'élevage.

Ce n'est qu'à l'occasion des séances de vaccination que les propriétaires viennent prêter main-forte à leurs bergers, expliquant ainsi que, même dans les populations d'agriculteurs, les occasions de contagion sont plus nombreuses pour les hommes. Car il est également coutume, après une séance de vaccination au cours de laquelle les efforts n'ont pas manqué, d'étan-

TABLEAU $\mathrm{N}^{\circ} \mathrm{V}$.-Comparaison des résultats allergologlques entre familles de bergers et d'agriculteurs des mêmes villages.

\begin{tabular}{|c|c|c|c|c|c|}
\hline \multirow[b]{2}{*}{ p r o s p e c té es } & \multicolumn{2}{|c|}{$\begin{array}{l}\text { Familles de } \\
\text { Bergers }\end{array}$} & \multicolumn{2}{|c|}{$\begin{array}{c}\text { Familles } \\
\text { d'Agriculteurs }\end{array}$} & \multirow{2}{*}{$o b s e r v a t i o n s$} \\
\hline & $\mathbf{T}$ & $\begin{array}{l}+ \text { Nombre } \\
\text { et p.100 }\end{array}$ & $\mathrm{T}$ & $\begin{array}{l}\text { + Nombre } \\
\text { et p.100 }\end{array}$ & \\
\hline $\begin{array}{l}\text { Banfora } \\
\text { (Haute-Volta) }\end{array}$ & 29 & $\begin{array}{l}10 \\
(34)\end{array}$ & 1239 & $\begin{array}{c}68 \\
(5,8)\end{array}$ & $\begin{array}{l}\text { Chi }^{2} \text { corrigé }=36,50 \\
\text { très significatif } R<0,0001\end{array}$ \\
\hline $\begin{array}{l}\text { Korhogo } \\
\text { (Côte d'Ivoire) }\end{array}$ & 54 & $\begin{array}{c}15 \\
(28)\end{array}$ & 1503 & $\begin{array}{c}118 \\
(7,9)\end{array}$ & $\begin{array}{l}\text { Chi }^{2} \text { corrigé }=24,03 \\
\text { très significatıf } R<0,0001\end{array}$ \\
\hline $\begin{array}{l}\text { Bouaké } \\
\text { (Côte d'Ivoire) }\end{array}$ & $B 3$ & $\begin{array}{l}23 \\
(28)\end{array}$ & 942 & $\begin{array}{c}50 \\
(5,3)\end{array}$ & $\begin{array}{l}\mathrm{Chi}^{2}=57, \mathrm{B9} \\
\text { très significatif } \mathrm{R}<0,00001\end{array}$ \\
\hline $\begin{array}{l}\text { Odienne } \\
\text { (Côte d'Ivoire) }\end{array}$ & 36 & $\begin{array}{c}13 \\
(36)\end{array}$ & 902 & $\begin{array}{c}54 \\
(6,0)\end{array}$ & $\begin{array}{l}\mathrm{Chi}^{2} \text { corrigé }=42,97 \\
\text { très significatif } \mathrm{R}<0,0001\end{array}$ \\
\hline Man & 23 & $\begin{array}{c}9 \\
(39)\end{array}$ & 766 & $\begin{array}{c}40 \\
(5,2)\end{array}$ & $\begin{array}{l}\text { Chi }^{2} \text { corrigé }=38,39 \\
\text { très significatif } R<0,0001\end{array}$ \\
\hline Total & 225 & $\begin{array}{c}70 \\
(31,1)\end{array}$ & 5352 & $\begin{array}{c}330 \\
(6,2)\end{array}$ & $\begin{array}{l}\text { Chi }^{2}=201,75 \\
\text { très significatif } R<0,000001\end{array}$ \\
\hline
\end{tabular}


cher sa soif en dégustant à la calebasse le lait fraîchement trait.

L'étude comparative à laquelle nous avons procédé dans les populations non pastorales, a montré qu'une différence hautement significative existe entre les bergers et leurs familles et le reste de la population : 31 p. 100 d'intradermoréactions positives dans le premier cas, et seulement 6 p. 100 dans le second.

\subsection{Variations entre les ethnies}

Dans les populations sahéliennes, l'ethnie joue un rôle prépondérant pour expliquer les différences des taux d'infection.

Les Peuls, véritables pasteurs, élèvent grands et petits ruminants; la traite est effectuée par les hommes ou par les femmes, et le lait (de vache) est consommé à l'état cru ou caillé.

Les Bellas ne pratiquent que l'élevage des chèvres et des ânes et consomment uniquement du lait de chèvre cru, la traite étant effectuée par les hommes.

Les Touaregs, bien que propriétaires de grands et petits ruminants, ne s'en occupent pas directement et en confient l'entretien aux Peuls, tout au moins en ce qui concerne les bovins et les ovins. Ils consomment le lait de vache souvent bouilli, mais parfois cru, la traite étant effectuée par les Peuls. Ils consomment également du lait de chamelle, qui leur est d'ailleurs exclusivement réservé, la traite étant effectuée par les Bellas ou par eux-mêmes.

Les Sonraïs, sédentaires, ne s'occupent pas directement des animaux qu'ils possèdent. Ce sont en effet, avant tout, des agriculteurs et ils en confient la garde et l'entretien à des bergers peuls. Lorsqu'ils consomment du lait, c'est sous la forme de lait caillé.

\subsection{Relations existant entre brucelloses humaine et animale et modes de transmission de la maladie à l'homme}

Il est évident que la brucellose humaine ne peut exister en l'absence de foyers animaux. Mais, comme nos enquêtes nous l'ont montré, il n'y a pas de liens entre les taux de l'endémie chez l'homme et chez l'animal. Les conditions écologiques, le mode de vie, les habitudes alimentaires des populations jouent en effet un tôle prépondérant.

Toutefois, dans les régions sahéliennes, nous avons pu faire les trois constatations suivantes:

1. Les villages les moins infestés du point de vue animal sont en général également les moins infestés du point de vue humain;

2. Les villages où la maladie animale est la plus importante ne sont pas nécessairement les plus touchés du point de vue humain;

3. Enfin, et réciproquement, les villages ayant présenté le plus de sujets positifs chez l'homme n'ont pas montré les taux les plus élevés chez les animaux.

En ce qui concerne la transmission de la maladie à l'homme, si le contact direct joue', à coup sûr, un rôle considérable, la transmission par le lait est également importante en Afrique, comme nous l'avons déjà précédemment souligné. En effet, celui-ci n'est que rarement consommé bouilli, mais le plus souvent à l'état cru ou caillé.

La relative fréquence des réactions positives, tant allergologiques que sérologiques, que nous avons observées chez de jeunes enfants qui, manifestement, n'ont pas encore eu de contacts directs avec les animaux, confirme cette hypothèse.

\section{CONCLUSION}

\section{Brucellose animale}

Les différentes enquêtes effectuées ont montré que la maladie existait dans toutes les régions prospectées et intéressait les différentes espèces animales, mais à des taux très variables selon les régions.

L'importance de l'endémie dans de nombreuses contrées, en particulier dans celles où de gros efforts sont entrepris pour promouvoir et développer l'élevage, doit inciter les autorités responsables des pays concernés à mettre en ceuvre sans tarder des mesures de prophylaxie médicale et sanitaire. Seule, l'application rigoureuse de celles-ci permettra d'enrayer l'extension de la maladie et d'en assurer le contrôle progressif avant d'en entreprendre ultérieurement l'éradication. 


\section{Brucellose humaine}

Les études réalisées ont montré le rôle essentiel des conditions écologiques dans l'étiologie de la brucellose humaine en Afrique. Hormis les bergers et leurs familles chargés de la garde des troupeaux en zone de savane, c'est, actuellement, essentiellement pour les populations pastorales du Sahel que la brucellose pose un grave problème de Santé Publique. Il est en effet à noter que dans les régions sahéliennes que nous avons prospectées, malgré une faible endémie animale, la maladie est fréquente dans la population humaine. Les médecins ayant exercé dans ces régions avaient déjà attiré notre attention sur les nombreux cas cliniques qu'ils avaient observés, mais pour lesquels ils n'avaient malheureusement pu avoir la confirmation du laboratoire, faute de moyens. Aussi, on ne peut qu'être alarmé sur les conséquences médicales que la brucellose doit avoir dans les populations des régions sahéliennes, lorsque l'endémie animale s'y situe à un niveau élevé.

\section{Remerciements}

Nous tenons particulièrement à remercier, pour l'aide ou les conseils qu'ils nous ont apportés :

- Monsieur le Professeur J. ROUX de l'U.E.R. de Médecine de Montpellier;

- Monsieur R. GAUMONT, Directeur de Recherches au Laboratoire Central de Recherches Vétérinaires de Maisons-Alfort;

- Messieurs les Directeurs de la Santé et de l'Elevage de Côte d'Ivoire, Haute-Volta et Niger;

- Messieurs les Médecins et Vétérinaires, Chefs de Service des régions prospectées ainsi que leur personnel;

- Monsieur le Dr J. BREMAN, du CDC Atlanta, Médecin Epidémiologiste à l'O.C. C.G.E.;

- Monsieur P. SALES, Adjoint au Chef du Service de Documentation et de Statistique de 1'O.C.C.G.E.

\section{RESUME}

Après un rappel très sommaire sur l'épidémiologie et la symptomatologie de la brucellose chez l'homme et chez l'animal, les auteurs relatent les conditions dans lesquelles ont été effectuées, par l'équipe BiologieZoonoses du Centre Muraz, dix enquêtes couplées, humaines et animales, pour étudier l'épidémiologie de cette affection en Afrique de l'Ouest: - Description succincte des différentes zones bioclimatiques prospectées, allant des régions sahéliennes dú Niger et du Nord de la Haute-Volta, aux régions forestières littorales du Sud de la Côte d'Ivoire.

- Méthodologic des enquêtes sur le terrain et au laboratoire.

Dans chacune des régions étudiées, une douzaine de villages en moyenne ont été prospectés.

Chez les animaux (bovins, caprins, ovins, ânes), trois tests ont été mis en couvre: un test sur le lait ou test de l'anneau (ring-test), pratiqué sur le terrain sur toutes les femelles laitières présentes (vaches, chèvres et brebis), et deux tests sérologiques réalisés au laboratoire à partir des échantillons de sérums recueillis sur un certain nombre d'animaux. 4108 ring-tests, dont 2907 chez les bovins, et 1225 sérums animaux furent étudiés.

Chez l'homme, dans chaque village, une centaine de personnes ont été choisies, par tirage au sort, après stratification préalable de la population en quatre groupes: garçons, filles, hommes et femmes. Trois tests également ont été utilisés : un test allergologique, l'intradermoréaction à la mélitine pratiquée sur le terrain, avec lecture le lendemain, et les deux tests sérologiques précédemment cités. 12739 intradermoréactions à la mélitine ont été pratiquées, mais seulement 10830 ont pu être contrôlées par suite d'un absentéisme variable selon les régions et les villages. 11990 sérums furent étudiés.

Chez les animaux, la maladie existe dans toutes les régions prospectées et intéresse les différentes espèces animales, mais à des taux très variables selon les régions. C'est dans l'espèce bovine que la maladie paraît la plus répandue. L'endémie semble de plus en plus importante au fur et à mesure que l'on descend vers le Sud (de $6 \mathrm{p}$. 100 de ring-tests positifs dans le Nord à 51 p. 100 dans le Sud chez les bovins). Dans le Nord, certains villages sont même apparus totalement indemnes ou très peu infectés, tandis que, dans le Sud, tous étaient contaminés et parfois à des taux très élevés (jusqu'à 100 p. 100 de ring-tests positifs et 63 p. 100 de sérologies positives). 
Chez l'homme, dans les régions de savane soudanienne et guinéenne et les zones forestières, peuplées d'agriculteurs ne s'occupant pas euxmêmes des animaux qu'ils possèdent, l'incidence de la maladie est peu importante en général, sauf chez les bergers et leurs familles. Par contre, dans les régions sahéliennes prospectées, malgré la faible endémie animale (6 à 8 p. 100 de ring-tests positifs chez les bovins), c'est là que furent observés les plus forts taux d'infection dans la population humaine (30 p. 100 d'intradermoréactions et 10 p. 100 de sérologies positives ă Dori). Ces faits qui semblent de prime abord paradoxaux, s'expliquent par les conditions écologiques: présence de populations en majorité à vocation pastorale, qui cohabitent étroitement avec leurs animaux, et pour lesquels la consommation de lait, à l'état cru ou caillé le plus souvent, est une des bases de l'alimentation.

L'étude statistique des résultats allergologiques chez l'homme a montré :

- une positivité significativement plus élevée chez les adultes que chez les enfants;

- une positivité significativement plus élevée chez les sujets de sexe masculïn que chez les sujets de sexe féminin, cette différence étant beaucoup plus marquée chez les adultes que chez les enfants;

- dans les régions à population non pastorale, une positivité significativement beaucoup plus élevée chez les bergers et leurs familles que dans le reste de la population;

- une différence significative entre certaines ethnies.

Enfin, les auteurs donnent quelques indications concernant les relations existant entre brucellose humaine et animale et les modes de transmission de la maladie à I'homme.

\section{SUMMARY}

Brucellosis in west Africa and its incidence in public health. Results of ten epidemiological surveys carried out in Ivory Coast,
Upper Volta and Niger from 1970 to 1973

A study of the epidemiology of human and animal brucellosis is presented, based on the findings from 10 separate investigations in West Africa. The study was carried out by a medical-veterinary team from the Centre Muraz, Bobo-Dioulasso. There is a brief review of the known epidemiology and clinical presentation of the disease in man and animals and a description of the area investigated and methodology used.

The study included zones ranging from the sahel of Niger and northern Upper Volta to the coastal forests of southern Iyory Coast. In each of the separate studies, a mean of 12 villages were investigated.

Three tests were used for the animals, which were cattle, goats, sheep and donkeys. The "ring-test" was done in the field on the milk of all lactating female animals (cows, she-goats and ewes). Two serologic tests were done at the laboratory. These were the seroagglutination test of Wright and the complement fixation test. 4108 ring-tests were performed of which 2907 were done on cattle. 1225 animal sera were studied.

For human studies, 100 persons were chosen at random in each village after preliminary sub-stratification of the population into four groups (boys, girls, men, women). Three diagnostic tests were used. The intradermal melitin skin test, a test of allergenicity, was performed in the field and read the day after administration. The two serologic tests used for humans were the same used for animals. Of 12739 intradermal tests given only 10830 were read; this was due to an absenteeism which varied in the different regions and villages. 11990 human sera were studied.

Brucellosis in animals was found in each region studied and affected all species at variable rates, according to region. The disease was found most prevalent among cattle. The prevalence increased as one moved south; 6 p. 100 ring-tests were positive in northern cattle and 51 p. 100 of cattle were positive in the south. Animals in certain northern villages appeared exempt from the disease or have a very low infection rate. In the south, the highest rates were observed; 100 p. 100 of ring-tests were positive in one village and 63 p. 100 of sera were positive in another village in the southern region.

Among humans, the prevalence of disease was lowest in the sudanian and guinean savanna and the forest. In these zones the agrarian population do not, themselves, tend to their own animals; the exception was the shepherd and his family. In the northern regions, despite the low 
animal endemicity ( 6 p. $100-8$ p. 100 positive ring-tests among cattle), the highest incidence of human infection was observed; 30 p. 100 intradermal reactions and 10 p. 100 of the serologic reactions were positive at Dori. These observations, seemingly paradoxical, are explained by understanding the local ecology. In this northern zone the population is essentially pastoral, living intimately with their animals. One of their dietary staples is milk, consumed either raw or curdled.

An analysis of the intradermal skin tests showed:

1. Adults significantly more positive than children;

2. Men significantly more positive than women. This difference was more marked among adults;

3. Shepherds and their families were significantly more infected in non-pastoral zones than others;

4. There were significant differences between efhnic groups.

The relationship between human and animal brucellosis and the mode of transmission of the disease to man are also considered.

\section{RESUMEN}

La brucelosis en Africa occidental y su incidencia sobre la salud pública. Resultados de diez encuestas epidemiológicas efectuadas en Costa de Marfil, Alta-Volta y Niger

Los autores sumariamente se acordan de la epidemiologia y de la sintomatologia de la brucelosis en el hombre y el animal. Indican, las condiciones en las cuales los investigadores de Biologia-zoonosis del Centro Muraz efectuaron diez encuestas juntadas, humanas y anumales, para estudiar la epidemiologia de dicha enfermedad en Africa del oeste:

- Descripcion sucinta de las diferentes zonas bioclimaticas exploradas, desde las regiones sahelianas del Niger y del norte de Alta-Voita hasta las regiones forestales litorales del sur de Costa de Marfil;

- Metodologia de las encuestas sobre terreno y en laboratorio.

Se exploraron unas doce aldeas por término medio en cada una de las regiones estudiadas.

En los animales (bovinos, cabrunos, ovinos, asnos) so hicieron tres pruebas: una prueba sobre la leche o prueba del anillo (ring-test) hecha en terreno en las hembras lecheras presentes (vacas, cabras y ovejas), y dos pruebas serológicas realizadas en laboratorio a partir de las muestras de sueros recogidos en un cierto número de animales. Se estudiaron 4108 ring-tests de los cuales 2907 en los bovinos, y 1225 sueros animales.

En lo concerniente el hombre, se elegieron por sorteo en cada aldea una centena de personas después de separación previa en cuatro grupos: muchachos, muchachas, hombres y mujeres. Se utilizaron tambien tres pruebas: una prueba alérgologica, la intradermoreacción con melitina hecha sobre terreno, con lectura el día siguiente y las dos pruebas serológicas ya citadas. Se hicieron 12739 intradermoreacciónes con melitina, pero solo se pudo comprobar 10830 como resultado de un absentismo variable según las regiones y las aldeas. Se estudiaron 11990 sueros.

En los animales, la enfermedad occurre en todas las regiones examinadas y ataca las diferentes especies con porcentajes varios según las regiones.

Es en los bovinos en los cuales la brucelosis es más frecuente. La endemia parece cada vez más importante a medida que se baja hacia el sur (de 6 p. 100 de ring-tests positivos en el norte hasta 51 p. 100 en el sur, en los bovinos).

En el norte, ciertas aldeas se encontraron totalmente indemnes o muy poco infectadas mientras que, en el sur, todas estaban contaminadas, a veces con porcentajes elevados (hasta 100 p. 100 de ring-tests positivos y 63 p. 100 de serologias positivas).

En el hombre, en las regiones de sabana sudanesa y guineana y en las zonas forestales, pobladas de agricultores no ocupandose ellos mismos de los animales que poseen, la incidencia de la infermedad generalmente es poco importante, excepto en los pastores y sus familias.

En cambio, en las regiones sahelianas examinadas, a pesar de la endemia animal poco importante (6 a 8 p. 100 de ring-tests positivos en los bovinos) es dónde se observaron los porcentajes de infección más importantes en el hombre. (30 p. 100 de intradermoreacción y 10 p. 100 de serologias positivas en Dori). Eso se explica por las condiciones ecologicas: presencia de poblaciones sobretodo pastorales, que cohabitan con sus animales y que consumen la leche cruda o el requesón.

El estudio estadístico de los resultados alergologicos en el hombre mostró : 
- una positividad significativamente más elevada en los adultos que en los muchachos;

- una positividad significativamente más elevada en los pacientes de sexo masculino que en los de sexo femenino, particularmente en lo concerniente a los muchachos;

- en las regiones con población no pastoral, una positividad significativamente mucho más elevada en los pastores y sus familias que en el resto de la población;

- una diferencia significativa entre ciertas étnias.

Al fín, se indican las relaciones entre la brucelosis humana y animal y los modos de transmisión de la enfermedad al hombre.

\section{BIBLIOGRAPHIE \\ (limitée aux techniques utilisées)}

1. ALTON (G. G.), LOIS M. JONES. La brucellose, technique de laboratoire. Genève, O.M.S., 1968. (Monographie O.M.S. n $^{\circ}$ 55).

2. AUBREVILLE (A.). Flore forestière soudanoguinéenne. Paris, Soc. d'Edit. Geog. Marit. et Colon, 1950, 523 p.

3. BEAUPERE (M.). Epizootiologie des brucelloses en Afrique noire francophone. Thèse doctorat vétérinaire, Alfort, 1966, $\mathrm{n}^{\circ} 44,91 \mathrm{p}$.
4. Comité Mixte F.A.O./O.M.S. d'experts de la brucellose, 1971. Cinquième rapport. Genève, O.M.S., 1971. (Sér. Rap. Techn. OM.S. no 464). 5. RENOUX (G.), GAUMONT (R.). Pathologie de la production du lait. II. Méthodes de diagnostic biologique des brucelloses animales. Paris, Cahier technique du Centre National de Coordination des Etudes et Recherches sur la Nutrition et l'Alimentation, 1966. 